\title{
Congenital lethal erythroderma
}

INSERM

\section{Source}

INSERM. (1999). Orphanet: an online rare disease and orphan drug data base. Congenital lethal erythroderma. ORPHA:1954

Congenital lethal erythroderma is a rare skin disorder characterized by erythrodermic, peeling skin from birth with no obvious nail or hair-shaft abnormalities and other associated anomalies including diarrhea, failure to thrive and severe hypoalbuminaemia resistant to correction by enteral or intravenous supplementation. An autosomal recessive mode of inheritance is highly probable. The prognosis is poor and infants die in the first months of life. There have been no further descriptions in the literature since 1992. 